\title{
CFD computation of flow in the flow path of a torque flow pump
}

\author{
Dmitry Svoboda ${ }^{1}$, Igor Borshchev ${ }^{1}$, Aleksandr Zharkovskii $^{1}$, Evgeniy Ivanov ${ }^{1}$ and Arsentiy \\ Klyuyev ${ }^{1, *}$ \\ ${ }^{1}$ Peter the Great St. Petersburg Polytechnic University, Polytechnicheskaya, 29, St. Petersburg, \\ 195251, Russia
}

\begin{abstract}
The results are presented of numerical and experimental research of fluid flow in the flow path of a torque flow pump with specific speed $n s \approx 55$. The $3 \mathrm{D}$ methods of CFD have been shown to allow for predicting energy characteristics of this type of pumps with a sufficient accuracy. According to the results of flow visualization the work process has been analysed and conclusions drawn to enhance TFP efficiency.
\end{abstract}

\section{Specific features of the work process of a torque flow pump}

Torque flow pumps (TFP) have been widely applied in treatment facilities and sewerage pump stations. This is because pumps of this type have a wide passage area and the flow passes mostly outside the impeller, and hard particles of admixtures barely touch its surfaces. Therefore, TFP are able to pump fecal, domestic and industrial wastewater containing fibrous and hard inclusions $[1,2]$. The TFP working process, however, based on the theory of turbulent jets and traces, the theory of vortex hydrodynamic lattices, is very complicated and not yet sufficiently studied, including with the use of modern 3D methods of numerical simulation $[3,4]$. There is no general theory of physical processes occurring in the flow path of the pump, and the publications suggest various hypotheses quite frequently contradicting each other [5]. In addition, the TFP efficiency is significantly lower than of conventional vane pumps.

An objective was set to try the methodology of hydrodynamic calculations of viscous fluid flow in the flow path of a torque flow pump on the basis of $3 \mathrm{D}$ methods of computational fluid dynamics (CFD). This will allow, at the next step of the research, the geometry of the flow path to be optimized in order to increase its efficiency, improve the cavitational and erosive qualities of the pump, and also the range of the best ratios of specific speed to be determined for TFP.

The research was done for a torque flow pump with Turo structural scheme that has a number of advantages against Wemco and Seka schemes and has therefore been most widely used [6].

Using a graphic editor of three-dimensional design SolidWorks the flow path with the specific speed $\mathrm{ns} \approx 55$ was designed. The vane system of the pump consists of an impeller with 12 single-stage radial vanes (Fig. 1).

\footnotetext{
* Corresponding author: arsentiyklyuyev@gmail.com
} 


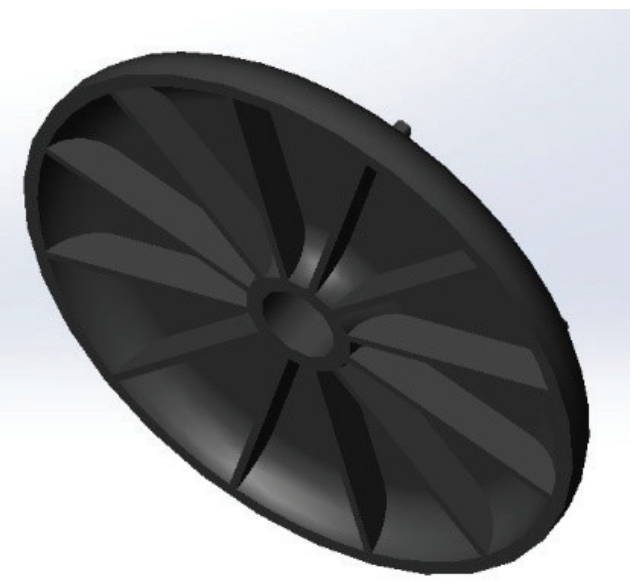

Fig. 1. Solid-body model of TFP impeller.

The computational region simulating the volume of fluid inside which the fluid flow being researched takes place included three domains: pump chamber, impeller and clearance (Fig. 2). Therewith the model took into account the effects of a radial clearance the relative value of which was rel $=/ D_{\text {RK }}=0.025$. A sufficiently high value of the clearance was chosen in order to rule out impeller jammings with hard inclusions and reduce possible abrasive wear.

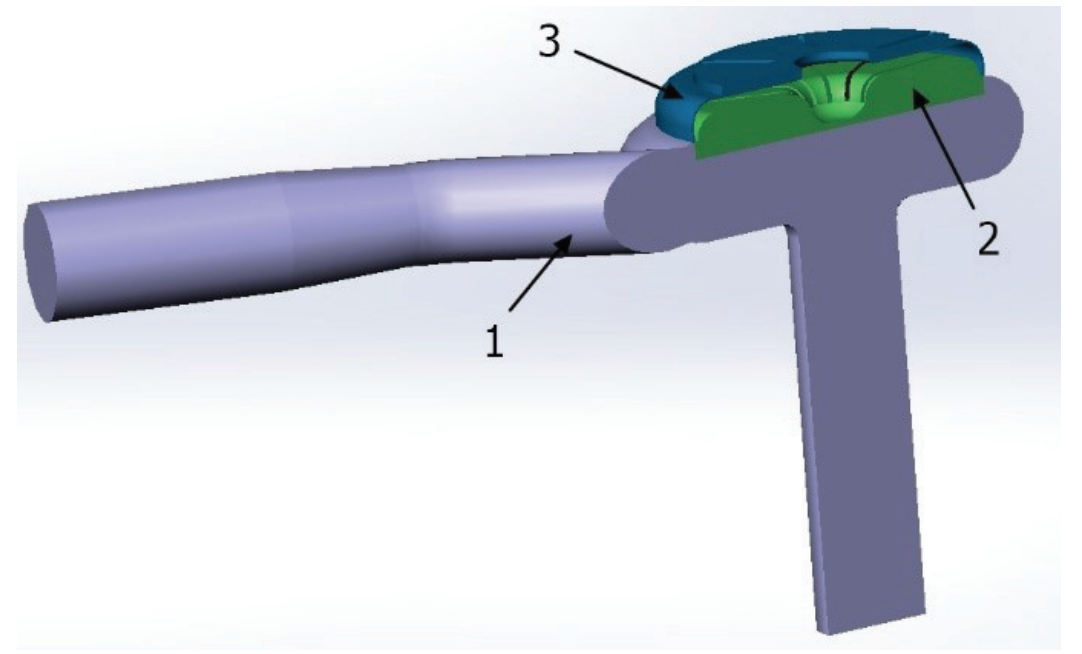

Fig. 2. Solid-body model of fluid flow: 1 - casing region, 2 - impeller region, 3 - clearance region.

\section{Simulation of the stationary state of the computational model}

Numerical research of flow in the flow path of TFP was done using the developed methodologies of supercomputer simulation on the basis of the modern methods of computational fluid dynamics (CFD), in the software package Ansys CFX on the heterogeneous cluster Polytechnic - RSK Tornado of the computational center of Saint Petersburg Polytechnic University. 
The computational region was discretized using a grid generator Ansys ICEM CFD. Unstructured grids having a thickening at sites with high gradients of local speeds were created. Also, near hard walls of the flow path prismatic layers were created the total thickness of which exceeded the thickness of the turbulent boundary layer. The number of the computational grid elements was: 2.7 million for impeller region, 4.5 million for pump chamber region, 2.5 million for clearance region. The grid quality was controlled using the built-in tools of ICEM CFD program. The grids were also controlled visually in order to rule out exceedingly stretched or skewed cells and to display problematic elements. Further on, the post-processor Ansys CFD-Post evaluated the profile of distribution of dimensionless parameter $\mathrm{y}^{+}$on all hard surfaces of the flow path.

When describing boundary conditions of such a complex and vortex-shaped flow as in the flow part of TFP, it is possible to apply different models of turbulence [7]. $n$ the first approximation, the calculation was performed in a stationary setup. And for closing of the Reynolds equations, two-parameter models were used: the high-Reynolds k- and the lowReynolds SST proposed by F. Menter. The Frozen Rotor condition was set on the interface surface between impeller domains and pump chamber. This condition means that the impeller is fixed in a certain angular position relative to the stator elements and no local averaging is performed.

At the inlet to the computational region the relative pressure of 1 bar was assigned, at the outlet - the mass rate corresponding to the calculated conditions. Thus, it was assumed that the outflow area was located sufficiently far from the regions with an intensive restructuring of the flow and the speed profile was distributed evenly on the area.

To facilitate the tracing and evaluation of the calculated integral parameters and visual control of their change at each iterative step the expressions were assigned which included the respective functions and arguments. The pump head $H$ was determined as a difference of static pressures at inlet and exit areas of the computational region, related to the density of the working fluid and the acceleration of gravity plus accretion of kinetic energy accounted for by the difference of the speeds in the outlet and inlet branches, without taking into account the residual swirl of the flow at the outlet from the pump.

The designed flow path (Fig. 2) was manufactured and tested on two different experimental benches on the base of industrial enterprises of St. Petersburg, which enabled verification of the results of the numerical simulation.

Fig. 3-4 present TFP dimensionless energy characteristics, which represent the dependence of the dimensionless head coefficient $K_{H}=H \cdot 3600 /\left(n^{2} \cdot D^{2}\right)$ and efficiency on the dimensionless flow coefficient $K_{Q}=Q \cdot 60 /\left(n \cdot D^{3}\right)$. 


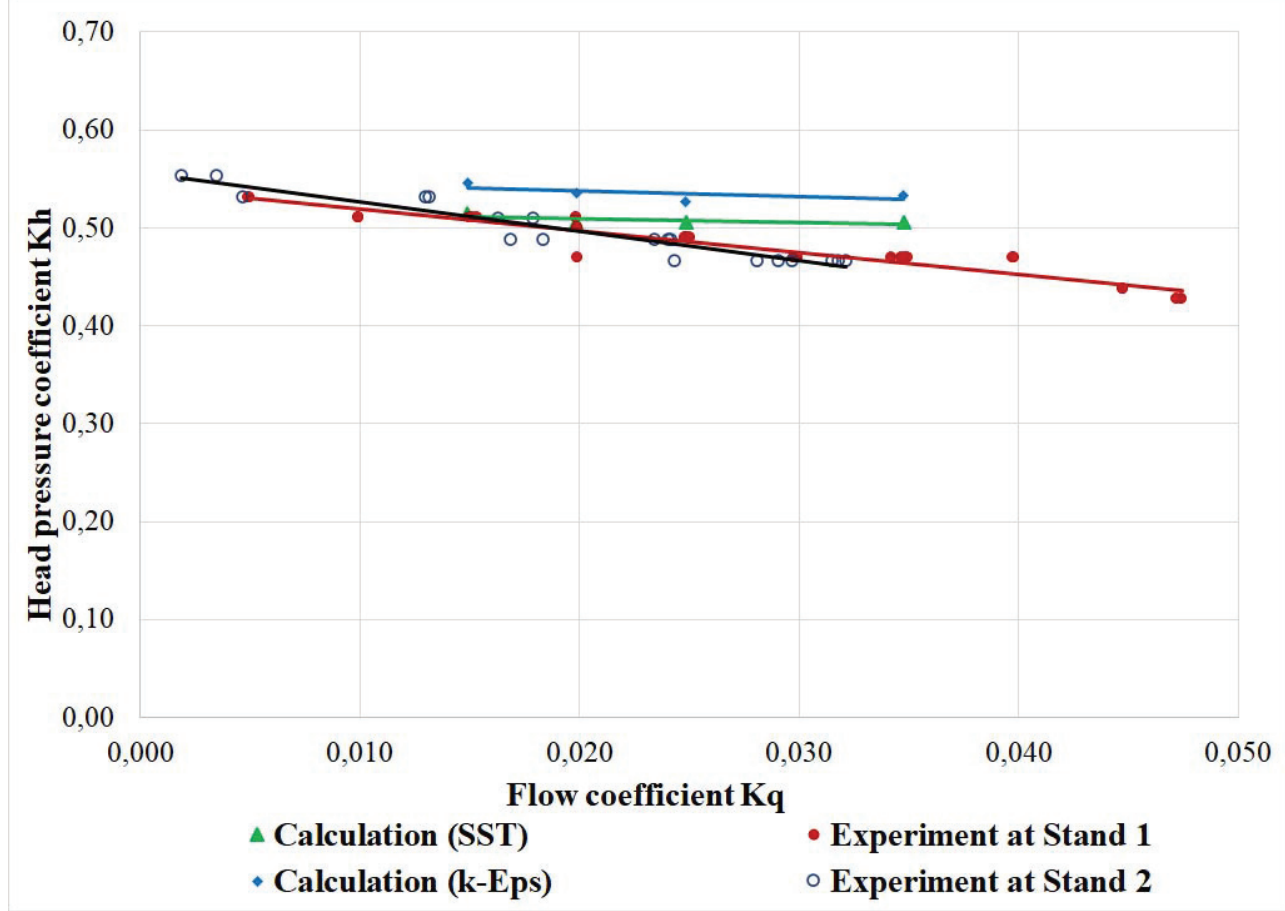

Fig. 3. Dimensionless pump properties.

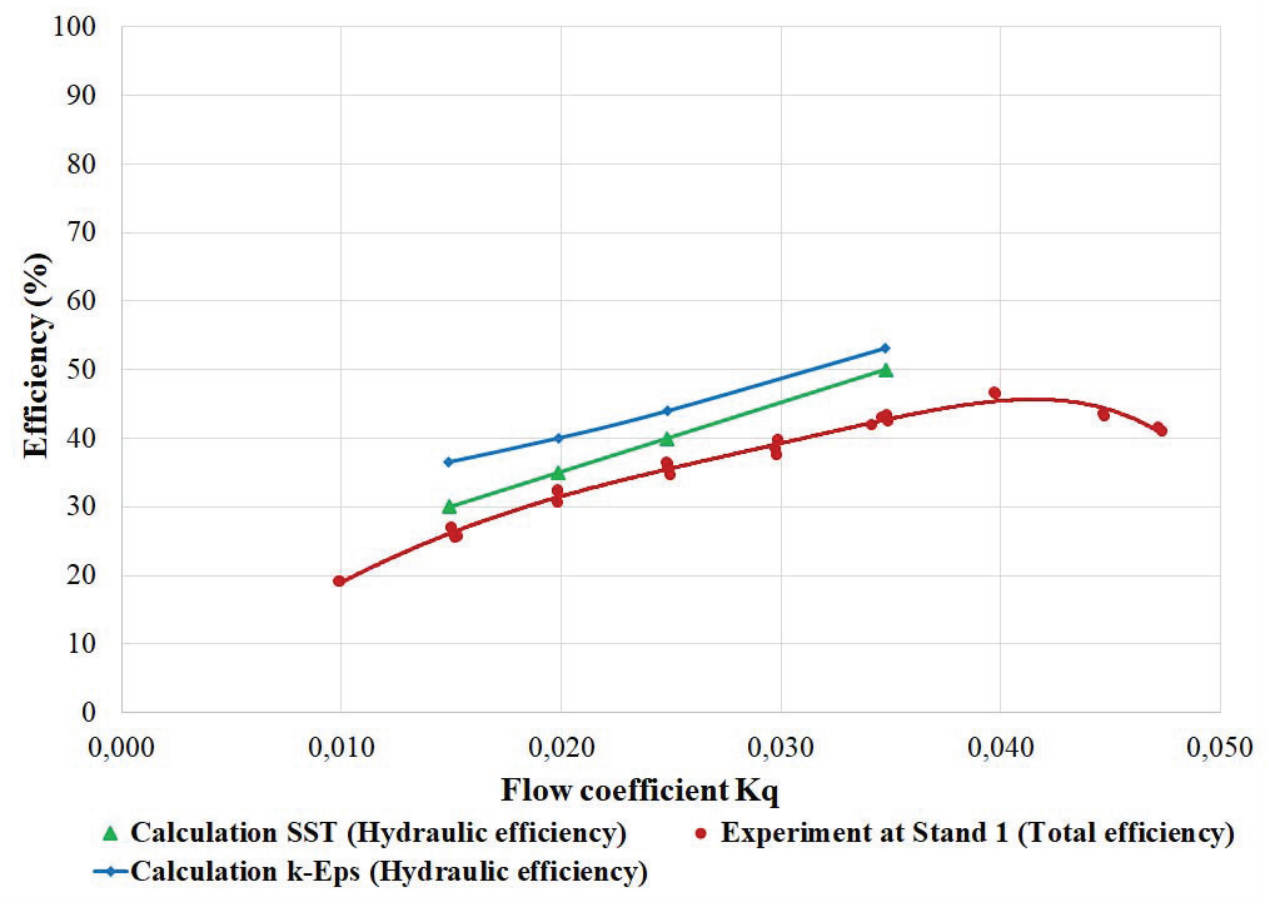

Fig. 4. Dimensionless efficiency properties. 
It can be seen from the graphs in Fig. 3 that the difference in the pressures obtained from the numerical computations with various turbulence models under the rated conditions $\mathrm{KQ}=0.025$ is $4 \%$.

The low-Reynolds SST turbulence model turned out to be more accurate, for it allows for a calculated characteristic that is better matched with the experimental one. The difference of the calculated and experimental pressure under the rated conditions does not exceed $3.5 \%$. This is to say that the suggested methodology is suitable for predicting the pressure on the entire range of TFP operation.

In evaluating the pump efficiency, it should be borne in mind that in calculating with CFD methods no mechanical losses in bearings or gland seals are determined. The difference of the graphs of the calculated hydraulic and total experimental efficiency under the rated conditions is $\sim 4 \%$. The losses in glands can be high, especially when they are too tightened, and have a significant effect of the total pump efficiency. Usually in first-order estimates the losses of power in glands and bearings are assumed to be 1-3\% depending on the pump output [8].

It should be borne in mind that a low calculated efficiency of $40 \%$ is characteristic for torque flow pumps, and the publications have conflicting hypotheses explaining this. S.S. Rudnev concluded, on the basis of analyzing TFP working process, in the article [9] that the theoretically achievable efficiency of such a process cannot exceed $50 \%$. The efficiency (40\%) obtained using 3D methods of CFD well agrees with the maximum efficiency $(41 \%)$ calculated by the formula (1) for torque flow pumps with Turo structural scheme suggested in the article [10]:

$$
\eta_{\max }=\frac{1}{-7.01 \cdot 10^{-3} \cdot \ln n_{s}+\frac{1.97}{n_{s}}+302.42 \cdot 10^{-6} \cdot n_{s}}
$$

It follows from the formula (1) that for the specific speed $\mathrm{ns} \approx 55$ the almost maximum possible pump efficiency is reached.

To evaluate the specifics of the TFP working process, the flow in its flow path was visualized.

\section{Flow visualization and analysis in the flow path of the pump}

As it is known, the working process of the free-vortex pump is fundamentally different from the working process of vane hydraulic machines, is very complicated, has not yet been sufficiently studied and currently has no established theory. Therefore, in addition to the determination of integral pump parameters, a flow analysis was performed in Ansys CFDPost on the basis of graphic visualization and post-processor processing of the obtained solution. Fig. 5-7 present the fields of speeds and pressures in various sections of the flow path under the rated conditions of operation of the pump being studied. 


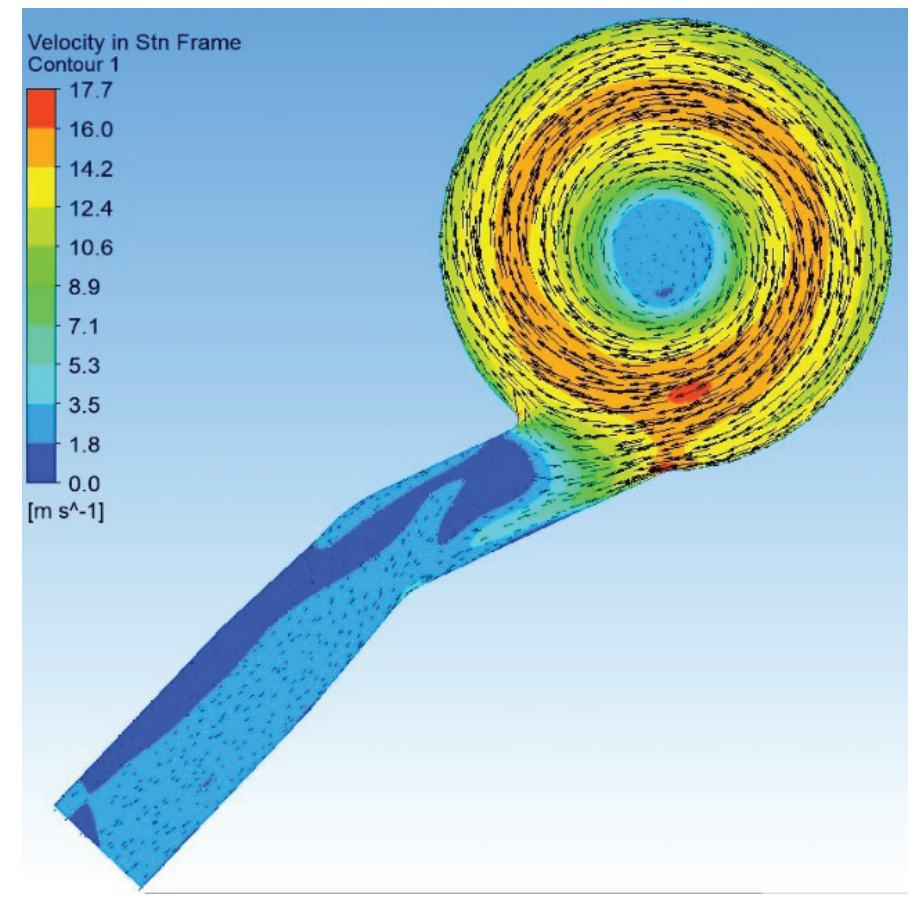

Fig. 5. Profile and field of vectors of absolute speed in cross section.

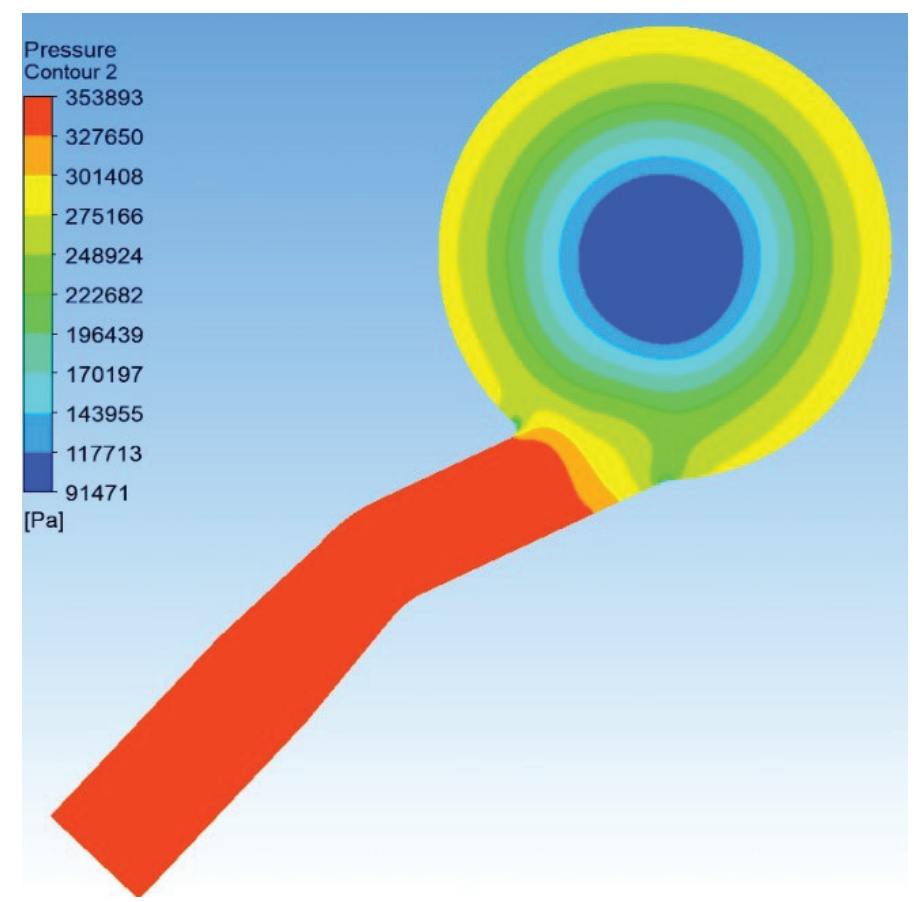

Fig. 6. Profile of static pressure in cross section. 


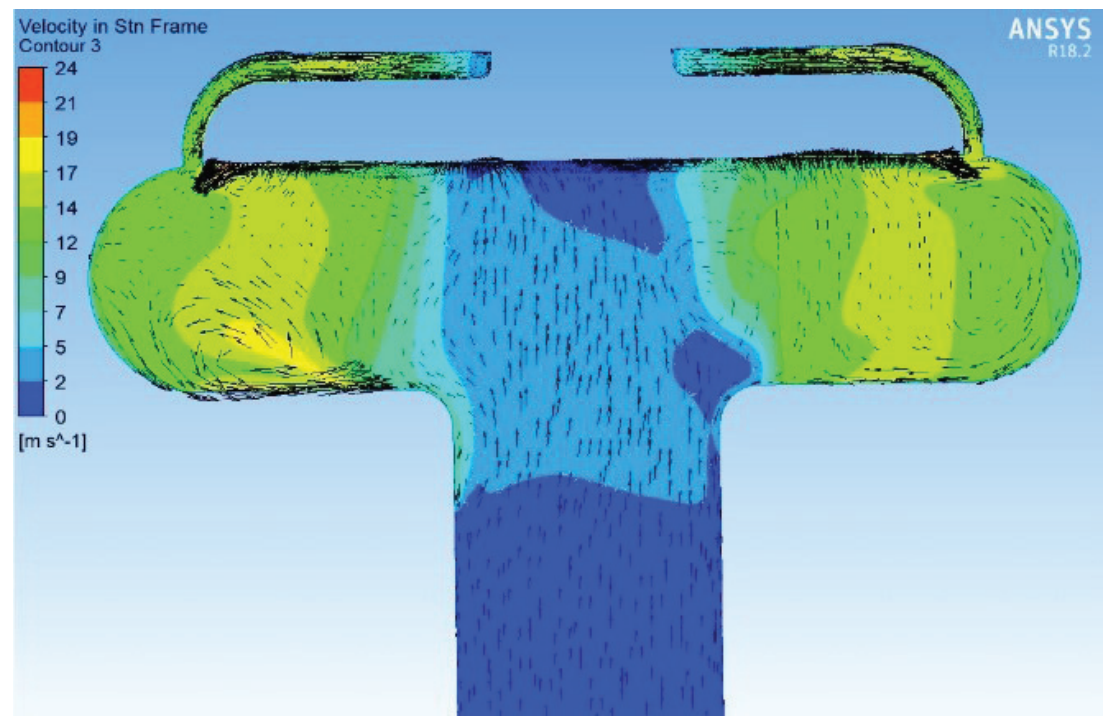

Fig. 7. Profile and field of vectors of absolute speed in longitudinal section.

It follows from the profiles in Fig. 5-7 that the working process in a torque flow pump is based on the transfer of energy from the impeller to a small amount of fluid in the form of a high-energy vortex flow. The vortex that has formed interacts with the basic pumped flow. Due to a turbulent energy exchange the low-energy basic flow receives energy from the high-energy vortex flow. The high-energy flow returns to the impeller where it replenishes its energy, and then the cycle is repeated.

Thus, the basic flow of the working fluid is spun into the casing of the pump by the impeller and moves under the action of centrifugal force spirally to the periphery (a vortex flow is created). The speed in the vortex attains $\mathrm{W}=17 \mathrm{~m} / \mathrm{s}$. The total flow energy is built up only on account of the increased speed energy of the working vortex flow of part of the fluid. The pressure in this type of vortex pump can be regulated on account of the rotational frequency of the impeller.

The transformation of the speed (kinetic) energy of the flow into static pressure takes place in the casing of the pump.

In the discharge pipe the flow moves unevenly. In some outlet branch sections the fluid is trapped by a reverse vortex flow directed toward the basic flow.

\section{Conclusions}

According to the results of the conducted research the following conclusions can be drawn:

Energy characteristics of a torque flow pump can be predicted by the results of the calculated fluid flow in its wet end on the basis of modern CFD 3D methods.

The analysis of the TFP working process has shown that the basic fluid flow passes outside the impeller, and the radial clearance in the casing of the pump should not have much effect on the pump head.

Accounting for a clearance in the computational scheme will increase the accuracy of determining hydraulic losses of disk friction, torque, and output of the pump, therefore it is advisable.

To describe the mathematical model of flow in the flow part of TFP, a low-Reynolds SST turbulence model is preferable. 
Whereas the working process depends on the interaction of the two flows, vortex and low-energy ones, it is possible to use in TFP a simplified impeller vane system in the form of radial plates.

Further improvement of the wet end requires optimization of the geometry of the pump chamber.

\section{References}

1. O. V. Baibakov, Vihrevye gidravlicheskie mashiny, (1981)

2. I. A. Kovalev, V. F. German, Svobodnovihrevye nasosy, (1990)

3. D. G. Svoboda, A. A. Zharkovskii, E. A. Ivanov, Chemical and Petroleum Engineering, 54, 673-680, (2019)

4. P. Sritram, R. Suntivarakorn, IOP Conference Series: Earth and Environmental Science, 257, (2019)

5. I. A. Kovalev, S. V Sapozhnikov, Gidromekhanika v inzhenernoj praktike, 59-60, (1996)

6. V. A. Solyanik, Visnik SumDU, 2, 146-149, (1998)

7. S. M. Belotserkovsky, A. S Ginevsky, Modelirovanie turbulentnyh struj i sledov na osnove metoda diskretnyh vihrej, 368, (1995)

8. A. N. Papyr, Osevye nasosy vodometnyh dvizhitelej, 252 (1965)

9. S. S. Rudnev, Tr. VNII gidromash, 43, 3-9 (1972)

10. V. O. Solyanik, Rabochij process $i$ energetichni yakosti vil'novihrovih nasosiv tipu “Turo”, 217, (1999) 